\title{
ON SEMISTABLE MORI CONTRACTIONS
}

\author{
YURI PROKHOROV
}

\begin{abstract}
We study Fano-Mori contractions with fibers of dimension at most one satisfying the semistability assumption. As an application of our technique we give a new proof of the existence of semistable 3 -fold flips.
\end{abstract}

\section{INTRODUCTION}

This paper is a continuation of our study of Mori contractions from threefolds to surfaces (see [12], [13], [14]). We refer to [10], [8] for the terminology of the minimal model theory.

Let $X$ be a normal algebraic threefold over $\mathbb{C}$ (or three-dimensional normal complex space) with only terminal singularities. A proper surjective morphism $f: X \rightarrow Z$ is called a Fano-Mori contraction if $f_{*} \mathcal{O}_{X}=\mathcal{O}_{Z}$ and the anticanonical divisor $-K_{X}$ is $f$-ample. Our interest is in the local structure of such contractions, so we shall always assume that $Z$ is not a point and $Z$ and $X$ are sufficiently small (algebraic or analytic) neighborhoods of some point $o \in Z$ and the fiber $f^{-1}(o)$, respectively. Note that we do not assume that $f$ is an extremal Mori contraction (i.e., $X$ is $\mathbb{Q}$-factorial and $\rho(X / Z)=1$ ). If the dimension of fibers of $f$ (near $f^{-1}(o)$ ) is at most one we can distinguish the following cases:

- $\operatorname{dim} Z=2$, then $f$ is called a Mori conic bundle,

- $\operatorname{dim} Z=3$ and $f$ contracts a divisor to a curve, then $f$ is called a 2-1-type divisorial contraction,

- $\operatorname{dim} Z=3$ and the exceptional locus of $f$ is one-dimensional, then $f$ is called a flipping contraction.

In this paper we deal with semistable Fano-Mori contractions which appear in the semistable minimal model program, see [19], [4], [18], [5], [8], and references therein.

Definition 1.1. A Fano-Mori contraction $f: X \rightarrow Z \ni o$ is said to be semistable if there exists an effective Cartier divisor $o \in T \subset Z$ such that $\left(X, f^{*} T\right)$ is divisorial log terminal (dlt).

It is clear that in the above definition we may replace $T$ with a general hyperplane section through $o$. In particular, in the case $\operatorname{dim} Z=3$ we 
may assume that $T$ is irreducible, normal, and $\left(X, f^{*} T\right)$ is purely log terminal (plt).

Example 1.2 ([12, Example 2.1]). The toric contraction $\left(\mathbb{P}^{1} \times\right.$ $\left.\mathbb{C}^{2}\right) / \boldsymbol{\mu}_{n}(0: a ; 1,-1) \rightarrow \mathbb{C}^{2} / \boldsymbol{\mu}_{n}(1,-1)$ is a semistable Mori conic bundle with $T=\left\{x_{1} x_{2}=0\right\} / \boldsymbol{\mu}_{n}$.

We shall show that the example above is very special: in "most" cases the surface $f^{-1}(T)$ is irreducible and normal.

Proposition 1.3. Let $f: X \rightarrow Z \ni$ o be a semistable Mori conic bundle and let $T$ be a general hyperplane section through $o$.

(i) If $T$ is reducible, then $f$ is analytically isomorphic to the Mori conic bundle from Example 1.2. In particular, $Z \ni o$ is a $D u$ Val point of type $A_{m-1}$.

(ii) If $T$ is irreducible, then $Z \ni o$ is smooth and the pair $\left(X, f^{*} T\right)$ is purely log terminal ( $p l t)$. In this case $S:=f^{*} T$ is a normal surface with cyclic quotient singularities of type $\mathrm{T}$ or Du Val of type $A_{n}$ (see $§ 3$ for the definition of T-singularities).

In case (ii) the structure of $X$ and $f$ is completely determined by the structure of the surface $S$ and the contraction $S \rightarrow T$. We study such contractions in $\S 5$.

In practice, it is very difficult to construct nontrivial examples of Mori conic bundles explicitly (cf. [12, §5]). Using deformation theory it is very easy to prove the existence (or non-existence) of semistable ones, see $\S 4$. In particular, we prove the following.

Theorem 1.4. For any three-dimensional terminal semistable singularity $U \ni P$, there is a semistable Mori conic bundle $f: X \rightarrow Z \ni o$ with a unique singular point which is analytically isomorphic to $U \ni P$.

The following result was inspired by M. Reid's "general elephant" conjecture (cf. $[12, \S 4]$ ) and provides an evidence for it.

Theorem 1.5 (cf. [3, Th. 0.4.5] [7, Th. 2.2], [18, Corr. 4.9]). Let $f: X \rightarrow Z \ni$ o be a semistable Fano-Mori contraction such that the dimension of fibers is at most one and let $T$ be a general hyperplane section through o. Then $K_{X}+f^{*} T$ is 1-complementary [15], i.e., there exists an effective integral Weil divisor $F$ such that $K_{X}+f^{*} T+F$ is log canonical (lc) and linearly trivial over $Z$. Moreover, $K_{X}+F$ is canonical and linearly trivial, the surface $F$ is normal, has only Du Val singularities of type $A_{n}$, and in the Stein factorization $F \rightarrow \bar{F} \rightarrow f(F)$, the same holds for $\bar{F}$. 
Using this theorem we give a new proof of the existence of semistable flips in $§ 7$. Our proof is based on Theorem 1.5, Kawamata's double covering trick [4], and the existence of certain canonical flops.

Terminology. The semistable MMP is originated in semistable degenerations of surfaces. Namely, let $h: \mathfrak{X} \rightarrow \Delta$ be a projective surjective morphism from smooth threefold to a smooth curve such that the general fiber is a smooth surface and special fibers are reduced simple normal crossings divisors. In this situation $\mathfrak{X} / \Delta$ satisfies the following property:

$$
\left(\mathfrak{X}, h^{*} P\right) \text { is dlt for every point } P \in \Delta .
$$

In order to obtain either a minimal or relative Fano model we run the $K$-MMP over $\Delta$. Every step of the $K$-MMP is in the same time a step of the $K+h^{*} P$-MMP. In particular, the property $(*)$ is preserved and all contractions and flips are semistable in our sense. This agrees more or less with definitions given in [19], [4], [1].

The semistability defined by Shokurov in [18] (cf. [11]) is also close to our one by [18, Lemma 1.4]. However the construction is inductive and given in terms of some (not necessarily projective) resolution.

Kollár and Mori [7, p. 541] defined semistable extremal neighborhoods $f: X \rightarrow Z \ni o$ in terms of general member $F_{Z} \ni\left|-K_{Z}\right|: f$ is semistable if $F_{Z} \ni o$ is a Du Val singularity of type $A_{n}$. By Theorem 1.5 our definition 1.1 implies Mori-Kollár's one. Conversely, if $F_{Z} \ni o$ is a Du Val singularity of type $A_{n}$, then $K_{X}+F+f^{*} T$ is $\log$ canonical (but not necessarily dlt) for some effective Cartier divisor $T$ on $Z$. Thus $f$ is "almost" semistable in our sense.

Our technique uses the Kawamata-Viehweg vanishing theorem, so the proofs work only in characteristic zero. The positive and mixed characteristic case was treated in [5].

Acknowledgments. I would like to thank Kyoto Research Institute for Mathematical Sciences for the hospitality during my stay there in 2002-2003. This paper is based on the subject of my talk given on the algebraic geometry seminar of RIMS on April 17, 2003. I am very grateful to the participants of this seminar for their attention and V. V. Shokurov for useful comments. The work was partially supported by the grant INTAS-OPEN-2000-269.

\section{Preliminary Results}

In this section we prove Proposition 1.3. 
Let $S$ be an algebraic surface (or two-dimensional complex space) having at worst quotient singularities and let $\varphi: S \rightarrow T$ be a contraction. We assume that $T$ is not a point and $T$ is a sufficiently small neighborhood of $o \in T$. We say that $\varphi$ is a $\log$ contraction if $-K_{S}$ is $\varphi$-ample. If furthermore any singularity of $S$ is of type $\mathrm{T}$ or $\mathrm{Du} \mathrm{Val}$, then we say that $\varphi$ is a T-contraction.

Lemma 2.1 (see [14, Lemma 2.5]). Let $f: X \rightarrow Z \ni$ o be a Mori conic bundle and let $T$ be an effective $\mathbb{Q}$-Weil divisor on $Z$ such that $\left(X, f^{*} T\right)$ is lc (resp. plt) at some point $P \in f^{-1}(o)$. Then $(Z, T)$ is $l c$ (resp. plt).

Lemma 2.2. Let $f: X \rightarrow Z \ni$ o be a semistable Mori conic bundle and let $T$ be an effective Cartier divisor such that $\left(X, f^{*} T\right)$ is dlt. If $T$ is irreducible, then $Z \ni o$ is smooth and for a general hyperplane section $o \in T_{\text {gen }} \subset Z$ the pair $\left(X, f^{*} T_{\text {gen }}\right)$ is plt.

Proof. Follows by Lemma 2.1 and Bertini's theorem.

Proposition 2.3. Let $f: X \rightarrow Z \ni$ o be a Mori conic bundle. Assume that there is an effective Weil divisor $T$ on $Z$ such that $\left(X, f^{*} T\right)$ is lc. If $T$ is reducible, then $f$ is analytically isomorphic to one of the following the Mori conic bundles

(i) $f$ from Example 1.2, or

(ii) $X^{\prime} / \boldsymbol{\mu}_{2}(1: 0: 0 ; 1,1) \rightarrow \mathbb{C}^{2} / \boldsymbol{\mu}_{2}(1,1)$, where $X^{\prime}=\left\{x_{0}^{2}+x_{1}^{2}+\right.$ $\left.x_{2}^{2} \phi(u, v)=0\right\} \subset \mathbb{P}_{x_{0}, x_{1}, x_{2}}^{2} \times \mathbb{C}_{u, v}^{2}$ and $\phi(u, v)$ is a $\boldsymbol{\mu}_{2}$-invariant without multiple factors, see [12, Example 2.3].

In particular, $Z \ni o$ is Du Val of type $A_{m-1}$. Moreover, the statement of Theorem 1.5 holds for $f$.

Proof. By Lemma 2.1, $T$ has exactly two components $T_{1}$ and $T_{2}$. Consider a base change

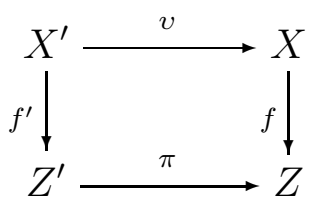

where $Z^{\prime}$ is smooth, $Z=Z^{\prime} / \boldsymbol{\mu}_{n}, X=X^{\prime} / \boldsymbol{\mu}_{n}$, and $\boldsymbol{\mu}_{n}$ acts on $Z^{\prime}$ free in codimension one (see [12, (1.9)]). Put $S=f^{*} T, S^{\prime}=v^{-1}(S)$, $T^{\prime}=\pi^{*}(T), T_{i}^{\prime}=\pi^{*}\left(T_{i}\right)$, and $S_{i}^{\prime}=f^{*} T_{i}^{\prime}$. Then $\left(X^{\prime}, S^{\prime}\right)$ is lc. Replacing $T_{1}^{\prime}$ and $T_{2}^{\prime}$ with general hyperplane sections, we may assume that $T_{1}^{\prime}$ is smooth and $\left(S_{1}^{\prime},\left.S_{1}^{\prime}\right|_{S_{2}^{\prime}}\right)$ is lc, where $\left.S_{1}^{\prime}\right|_{S_{2}^{\prime}}$ is a Cartier divisor on $S_{1}^{\prime}$. In this situation, $S_{1}^{\prime}$ has at worst Du Val singularities. Hence $X^{\prime}$ is Gorenstein. Since $f^{\prime-1}\left(o^{\prime}\right)$ is reduced, by $[12, \S 2] X / Z$ we have only 
two choices for the action of $\boldsymbol{\mu}_{n}$. Finally, the statement of Theorem 1.5 easily follows by the proposition below.

Proposition 2.4 (see [15, Prop. 4.4.1]). Let $f: X \rightarrow Z \ni$ o be a contraction and let $S$ be a reduced divisor on $X$ such that $S \cap f^{-1}(o) \neq$ $\varnothing,(X, S)$ is plt and $-\left(K_{X}+S\right)$ is $f$-nef and $f$-big. If $K_{S}+\operatorname{Diff}_{S}$ is $n$-complementary, then so is $K_{X}+S$. Here $\operatorname{Diff}_{S}$ is the different, a correcting term in the Adjunction Formula $K_{S}+\operatorname{Diff}_{S}=\left.\left(K_{X}+S\right)\right|_{S}$, see $[10$, Ch. 16].

From now on we consider semistable Fano-Mori contractions $f: X \rightarrow$ $Z \ni o$ such that $\left(X, f^{*} T\right)$ is plt (and the dimension of fibers is at most one).

\section{Singularities of Class T}

Let $\boldsymbol{\mu}_{n}$ acts on $\mathbb{C}^{2}$ via $(x, y) \rightarrow\left(\eta^{a} x, \eta^{b} y\right)$, where $\eta$ is a primitive $n$th root of unity and $\operatorname{gcd}(n, a)=\operatorname{gcd}(n, b)=1$. In this case we say that the quotient $\mathbb{C}^{2} / \boldsymbol{\mu}_{n}$ is a singularity of type $\frac{1}{n}(a, b)$. This singularity can be written as $\frac{1}{n}(1, q)$, so it is determined by the fraction $n / q$. The minimal resolution of $\frac{1}{n}(1, q)$ can be described as follows. The dual graph of the exceptional divisor is a chain of smooth rational curves whose self-intersections $-b_{1}, \ldots,-b_{\varrho}$ are determined by the continued fraction expansion

$$
\frac{n}{q}=b_{1}-\frac{1}{b_{2}-\frac{1}{\cdots \frac{1}{b_{\varrho}}}} .
$$

For typographical reasons we denote the fraction in $(3.1)$ by $\left[b_{1}, \ldots, b_{\varrho}\right]$. Put $\varrho(n / q):=\varrho$. Define also the following invariants:

- $\iota(n / q)=n / \operatorname{gcd}(n, q+1)$, the index of $\frac{1}{n}(1, q)$,

- $\beta(n / q)=\operatorname{gcd}(n, q+1) / \iota(n / q)=\operatorname{gcd}(n, q+1)^{2} / n$,

- $\gamma(n / q)=(q+1) / \operatorname{gcd}(n, q+1)$.

By definitions, $\iota, \gamma \in \mathbb{N}, \gamma \leq \iota, \operatorname{gcd}(\iota, \gamma)=1$. Thus we have the triple $(\iota, \beta, \gamma)$ which determines $n / q$ :

$$
n=\beta \iota^{2}, \quad q=\beta \iota \gamma-1,
$$

Note that presentation of a cyclic quotient singularity in the form $\frac{1}{n}(1, q)$ is not unique: $\frac{1}{n}\left(1, q^{\prime}\right)$ defines the same singularity if and only if either $q \equiv q^{\prime}$ or $q q^{\prime} \equiv 1 \bmod n$. Clearly, $\varrho(n / q)=\varrho\left(n / q^{\prime}\right)$. Since $\operatorname{gcd}(n, q+1)=\operatorname{gcd}\left(n, q^{\prime}+1\right)$, we have $\beta(n / q)=\beta\left(n / q^{\prime}\right)$ and $\iota(n / q)=\iota\left(n / q^{\prime}\right)$. 
Definition 3.2. A cyclic quotient non-Du Val singularity $\frac{1}{n}(a, b)$ is said to be of type $\mathrm{T}$ (or simply T-singularity) if $(a+b)^{2} \equiv 0 \bmod n$. (It is easy to see that this definition does not depend on the representation in the form $\left.\frac{1}{n}(a, b)\right)$.

If $\frac{1}{n}(1, q)$ is a T- (resp. Du Val) singularity, then we say that $n / q$ is a T- (resp. Du Val) fraction and $\left[b_{1}, \ldots, b_{\varrho}\right]$ is T- (resp. Du Val) chain. Thus $n / q$ is a T-fraction if and only if $\beta(n / q) \in \mathbb{Z}$.

Lemma 3.3. Let $q q^{\prime} \equiv 1 \bmod n$. Then $n / q$ is a T-fraction if and only if $q+q^{\prime}=n-2$ if and only if $\gamma\left(n / q^{\prime}\right)+\gamma(n / q)=\iota(n / q)$.

Remark 3.4. If $\iota(n / q)=2$, then $\gamma(n / q)=1$ and $n / q$ is a T-fraction. It is easy to see that this implies either

$$
n / q=[4], \quad \text { or } \quad n / q=[3,2, \ldots, 2,3] .
$$

Moreover, $\beta(n / q)=\varrho(n / q)$. Conversely, any chain such as in (3.5) has $\iota=2$.

The minimal resolutions of T-singularities are completely described.

Proposition $3.6([9])$. (i) If the chain $\left[b_{1}, \ldots, b_{\varrho}\right]$ is of type $\mathrm{T}$, then so are the chains

$$
\text { a) } \left.\left[2, b_{1}, \ldots, b_{\varrho}+1\right] \text { and } b\right) \quad\left[b_{1}+1, \ldots, b_{\varrho}, 2\right]
$$

(ii) Every T-chain can be obtained by starting with one of the chains (3.5) and iterating the steps described in (i).

For a chain $\left[b_{1}, \ldots, b_{\varrho}\right]$, we denote corresponding log discrepancies by $\alpha_{1}, \ldots, \alpha_{\varrho}$.

Lemma 3.7. In the above notation one has $\alpha_{1}=(q+1) / n=\gamma / \iota$. If $n / q$ is a T-fraction, then $\alpha_{\varrho}=1-\gamma / \iota$.

Proof. The $\frac{1}{n}(1, q)$-weighted blow-up gives us the first relation. The second one follows by Lemma 3.3.

Corollary 3.8. Let $\left[b_{1}, \ldots, b_{\varrho}\right]$ be any chain. The following are equivalent:

(i) $\left[b_{1}, \ldots, b_{\varrho}\right]$ is of type $\mathrm{T}$;

(ii) $\alpha_{1}+\alpha_{\varrho}=1$.

Theorem 3.9 ([2, Prop. 5.9], [9]). Let $S \ni P$ be a germ of a twodimensional quotient singularity. The following are equivalent:

(i) $S \ni P$ is either Du Val or of type $\mathrm{T}$,

(ii) $S \ni P$ has a $\mathbb{Q}$-Gorenstein one-parameter smoothing, 
(iii) there is a terminal three-dimensional singularity $X \ni P$ and an embedding $P \in S \subset X$ such that $S \subset X$ Cartier at $P$ and $(X, S)$ is plt.

\section{Constructing semistable Mori conic Bundles via DEFORMATIONS}

In this section all varieties are assumed to be analytic spaces.

Definition 4.1. A $\log ($ resp. T) contraction $\varphi: S \rightarrow T \ni o$ with $\operatorname{dim} T=1$ is called a $\log ($ resp. T) conic bundle.

Theorem 4.2. Let $\varphi: S \rightarrow T \ni o_{T}$ be a T-conic bundle. There exists a semistable Mori conic bundle $f: X \rightarrow Z \ni o_{Z}$ with smooth base and embeddings

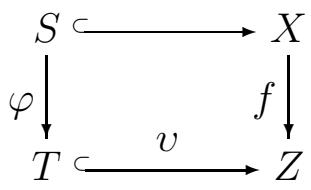

such that $v\left(o_{T}\right)=o_{Z}$ and $(X, S)$ is plt.

We shall construct $X$ as an one-parameter family of $T$-contractions.

Proof. We replace $S$ and $T$ with their compactifications so that $S$ and $T$ are projective, $T \simeq \mathbb{P}^{1}$, and $\varphi: S \rightarrow T$ is smooth outside of $\varphi^{-1}\left(o_{T}\right)$. Let $P_{i}$ be singular points of $S$.

Denote $\operatorname{Def}(S)$ (resp. $\left.\operatorname{Def}\left(S, P_{i}\right)\right)$ the base space of the versal deformation of $S$ (resp. of the singularity $S \ni P_{i}$ ). Let $s: \mathcal{S} \rightarrow \operatorname{Def}(S)$ be the versal family. Thus we may assume that $S=s^{-1}(o)$ for some $o \in \operatorname{Def}(S)$.

From [7, Proposition 11.4] we obtain the existence of the diagram of morphisms of complex analytic spaces.

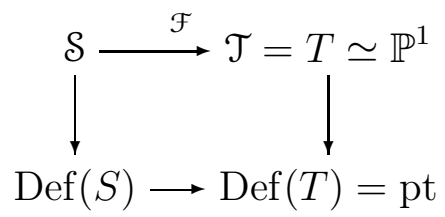

where $\mathcal{F}(S)=T$ and $\left.\mathcal{F}\right|_{S}=\varphi$.

There is a natural (pull-back) morphism of germs of analytic spaces

$$
\operatorname{Def}(S) \longrightarrow \prod_{i} \operatorname{Def}\left(S, P_{i}\right)
$$

The obstruction to globalize deformations in $\prod_{i} \operatorname{Def}\left(S, P_{i}\right)$ lies in $R^{2} \varphi_{*} \Theta_{S}$, where $\Theta_{S}=\left(\Omega_{S}^{1}\right)^{\vee}$, the tangent sheaf of $S$. Since $R^{2} \varphi_{*} \Theta_{S}=$ 
0, the map (4.3) is smooth. In particular, every deformation of singularities $S \ni P_{i}$ may be globalized (cf. [7, 11.4.2]).

By Theorem 3.9 every singularity of $S$ admits a $\mathbb{Q}$-Gorenstein oneparameter smoothing. Therefore there is a smoothing $g: X \rightarrow \Delta \ni 0$, where $g^{-1}(0)=S, X$ is $\mathbb{Q}$-Gorenstein and $\Delta \subset \mathbb{C}$ is a small disc. By Inversion of Adjunction $(X, S)$ is plt and since $S$ is Cartier, $X$ has at worst terminal singularities near $S$.

Put $Z=T \times \Delta$ and let $f: X \rightarrow Z$ be the projection. It is clear that $\left.f\right|_{S}=\varphi: S \rightarrow T$. Therefore $-K_{X}$ is $f$-ample near $S$. Shrinking $Z$ we get a Mori conic bundle $f: X \rightarrow Z \ni o=\left(o_{T}, 0\right)$.

\section{TWO-DIMENSIONAL LOG CONTRACTIONS}

Notation 5.1. Let $\varphi: S \rightarrow T \ni o$ be a log contraction. We assume that $S$ has at least one non-Du Val singularity. Let $\mu: \tilde{S} \rightarrow S$ be a minimal resolution and let $\phi: \tilde{S} \rightarrow T$ be the composition map. Take an effective Cartier divisor $D$ on $S$ such that $\operatorname{Supp}(D)=\varphi^{-1}(o)$ and $-D$ is $\varphi$-nef. For example, in the case $\operatorname{dim} T=1$ we can put $D=\varphi^{*}(o)$ (the scheme-theoretical fiber) while in the case $\operatorname{dim} T=2$ we can put $D:=\varphi^{*} \varphi_{*} H-H$, where $H$ is a very ample divisor on $S$ such that $\varphi_{*} H$ is Cartier.

One can write the standard formula

$$
\mu^{*} K_{S}=K_{\tilde{S}}+\Delta
$$

where $\Delta$ is an effective exceptional divisor, so-called, codiscrepancy divisor. Since the singularities of $S$ are log terminal, $\lceil\Delta\rceil=0$. We also write $\mu^{*} D=\sum l_{i} L_{i}+e_{j} E_{j}$, where the $E_{i}$ (resp. $\left.L_{i}\right)$ are $\mu$-exceptional (resp. non- $\mu$-exceptional) components and $l_{i}, e_{i} \in \mathbb{N}$. Put $L=\sum l_{i} L_{i}$ and $E=e_{j} E_{j}$. Thus, $D=\mu_{*} L$ and $\operatorname{Supp}(\Delta) \subset \sum E_{i}$.

Lemma 5.3. Notation as above.

(i) $\operatorname{Supp}(L+E)$ is a tree of smooth rational curves;

(ii) all the components of $L$ are $(-1)$-curves;

(iii) $\Delta \cdot L_{i}<1$ for all $i$;

(iv) if $\operatorname{dim} T=1$, then $L \cdot \Delta+2=\sum l_{i}$.

Proof. (i) is obvious because $\phi$ is flat in the case $\operatorname{dim} T=1$ and because $Z \ni o$ is a rational singularity in case $\operatorname{dim} T=2$. By (5.2) we have

$$
0>\mu^{*} K_{S} \cdot L_{i}=K_{\tilde{S}} \cdot L_{i}+\Delta \cdot L_{i}=\Delta \cdot L_{i}-2-L_{i}^{2} .
$$

Since $L_{i}^{2}<0$ and $\Delta \cdot L_{i} \geq 0$, we have $L_{i}^{2}=K_{\tilde{S}} \cdot L_{i}=-1$ and $\Delta \cdot L_{i}<1$. This shows (ii) and (iii). For (iv) we note that $\mu^{*} K_{S} \cdot L=-2$. Thus,

$$
\sum l_{i}=-K_{\tilde{S}} \cdot L=-\mu^{*} K_{S} \cdot L+\Delta \cdot L=\Delta \cdot L+2 .
$$


Remark 5.4. $\quad$ (i) It is easy to see that the condition (iii) of 5.3 is also sufficient. Assume that conditions 5.1 hold except for the ampleness of $-K_{S}$. If $\Delta \cdot L_{i}<1$ for all $i$, then $\varphi$ is a $\log$ contraction, i.e., $-K_{S}$ is ample.

(ii) If $S$ has a unique non-Du Val point, then (iii) holds.

To describe log contractions we make use the weighted graph language. By a weighted graph $\Gamma$ we mean a graph where each vertex is given a weight $b_{i} \geq 1$. With a weighted graph $\Gamma=\left\{v_{1}, \ldots, v_{\varrho}\right\}$ we associate a quadratic form by setting $v_{i}^{2}=-b_{i}$ and $v_{i} \cdot v_{j}$ for $i \neq j$ is equal to the number of edges joining $v_{i}$ and $v_{j}$. We say that a weighted graph $\Gamma=\left\{v_{1}, \ldots, v_{\varrho}\right\}$ is elliptic (resp. parabolic) if the associated quadratic form has signature $(0, \varrho)$ (resp. $(0, \varrho-1))$. Vertices with $b_{i}=1$ will be referred to (and drawn) as black vertices those with $b_{i} \geq 2$ as white. Weights $b_{i}=1$ and $b_{i}=2$ will be omitted.

By the blow-up of a vertex $v_{i}$ we mean the following transformation: the weight of the vertex $v_{i}$ increases by one, that is, $b_{i}^{\prime}=b_{i}+1$ and a new black vertex is added to the graph, attached by an edge to the vertex $v_{i}^{\prime}$. Similarly the blow-up of an edge $\left\{v_{i}, v_{j}\right\}$ is the following transformation: the weight of the vertices $v_{i}$ and $v_{j}$ increase by one, the number of edges joining $v_{i}$ and $v_{j}$ decreases by one, and a new black vertex is added to the graph, attached by edges to the vertices $v_{i}^{\prime}$ and $v_{j}^{\prime}$. The inverse transformations are called contractions. One can easily see how the above transformations are related to blow-ups of curves on a smooth surface.

We denote by $\left[a_{1}, \ldots, a_{r}\right]$ a (weighted) chain and by $\left[p\left|a_{1}, \ldots, a_{r}\right|\right.$ $\left.b_{1}, \ldots, b_{s} \mid c_{1}, \ldots, c_{l}\right]$ a fork $\Gamma$ having the central vertex $v_{0}$ of weight $p$ so that $\Gamma \backslash\left\{v_{0}\right\}$ is a disjoined union of chains $\left[a_{1}, \ldots, a_{r}\right],\left[b_{1}, \ldots, b_{s}\right]$, and $\left[c_{1}, \ldots, c_{l}\right]$, where vertices corresponding $a_{1}, b_{1}$, and $c_{1}$ are adjacent to $v_{0}$.

Now in notation 5.1 we denote by $\Gamma(\varphi)$ the dual graph of the fiber $\phi^{-1}(o)$. By (i) of Lemma 5.3, $\Gamma(\varphi)$ is a tree. Moreover, the graph $\Gamma(\varphi)$ is parabolic whenever $\operatorname{dim} T=1$ and elliptic whenever $\operatorname{dim} T=2$.

Lemma 5.5. The fork $[1|a| b \mid c]$ is not elliptic for $a, b, c \geq 1$. The following graphs are parabolic (and not elliptic):

(i) chains $[1,1],[1,2, \ldots, 2,1],[2,1,2]$,

(ii) the fork $[2|2| 2 \mid 2, \ldots, 2,1]$

Corollary 5.6. Let $D_{i}$ be irreducible components of $D$, then

(i) intersection points $D_{i} \cap D_{j}$ are singular and not Du Val,

(ii) there are at most two singular points on every $D_{i}$, 
(iii) $\left(S, D_{i}\right)$ is plt near every Du Val point,

(iv) $S$ has no Du Val singularities of type $D_{n}$ and $E_{n}$.

\section{Configuration of singular points.}

Lemma 5.7. Let $\varphi: S \rightarrow T \ni$ o be a T-contraction and let $C$ be a component of $D$. Assume that $C$ contains exactly two singular points of $S$ and they are of type $\mathrm{T}$ (not Du Val). Then $(S, C)$ is plt.

Proof. If $C \neq \operatorname{Supp}(D)$, then $C$ is contractible over $T$, i.e., there is a decomposition $S \rightarrow T^{\prime} \rightarrow T$ such that $\varphi^{\prime}: S \rightarrow T^{\prime}$ is birational and $C$ is the only $\varphi^{\prime}$-exceptional divisor. Replacing $T$ with $T^{\prime}$ we may assume that $C=\operatorname{Supp}(D)$.

Let $P_{1}, P_{2}$ be singular points. Assume that $(S, C)$ is not plt near $P_{1}$. We claim that $(S, C)$ is plt near $P_{2}$. Indeed, take two general hyperplane sections $H_{1}$ and $H_{2}$ passing through $P_{1}$ and $P_{2}$. For some $0<\varepsilon^{\prime} \ll \varepsilon \ll 1$ the divisor $-\left(K_{S}+\left(1-\varepsilon^{\prime}\right) C+\varepsilon H_{1}+\varepsilon H_{2}\right)$ is $\varphi$-ample and the pair $\left(S,\left(1-\varepsilon^{\prime}\right) C+\varepsilon H_{1}+\varepsilon H_{2}\right)$ is not lc at $P_{1}$ and $P_{2}$. This contradicts Connectedness Lemma [17, 5.7].

Thus $(S, C)$ is plt near $P_{2}$ and $\Gamma(\varphi)$ has the form

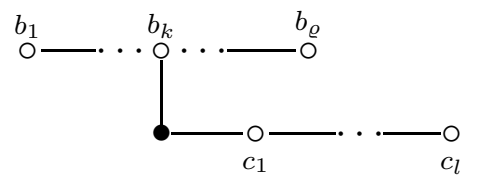

where $\left[b_{1}, \ldots, b_{\varrho}\right]$ and $\left[c_{1}, \ldots, c_{l}\right]$ are T-chains (i.e. $\Gamma(\varphi)=\left[b_{k}\right.$ $\left.\left.b_{k-1}, \ldots, b_{1}\left|b_{k+1}, \ldots, b_{\varrho}\right| c_{1}, \ldots, c_{l}\right]\right)$. Let $\alpha_{1}^{\prime}$ and $\alpha_{k}$ be log discrepancies of the vertices corresponding to $c_{1}$ and $b_{k}$, respectively. By Lemma 5.3 we have $\alpha_{1}^{\prime}+\alpha_{k}>1$. Let

$$
\Gamma(\varphi)=\Gamma_{0} \rightarrow \Gamma_{1} \rightarrow \cdots \rightarrow \Gamma_{r}=\Gamma^{\prime}
$$

be the sequence of contractions of black vertices. If $b_{k}=2$, then $\Gamma_{1}$ contains the fork $\left[1\left|b_{k-1}\right| b_{k+1} \mid c_{1}-1\right]$. This contradicts Lemma 5.5. Therefore $b_{k} \geq 3$. The same arguments show that in each graph $\Gamma_{i}$ the central vertex (corresponding to $b_{k}$ ) is not black. Since $\left[b_{1}, \ldots, b_{\varrho}\right]$ is a chain of type $\mathrm{T}$, we may assume that $b_{\varrho}=2$ and $b_{1} \geq 3$. Thus

$$
\Gamma^{\prime}=\left[b_{k}-s\left|b_{k-1}, \ldots, b_{1}\right| b_{k+1}, \ldots, b_{\varrho} \mid c_{s}-1, c_{s+1}, \ldots, c_{l}\right],
$$

where $s \geq 1, b_{k}-s \geq 2$, and $c_{s}-1 \geq 2$. Clearly, $\Gamma^{\prime}$ is elliptic and $\log$ terminal (because $-\left(K_{\tilde{S}}+\Delta\right)$ is nef and big over $T$ ). Now we use the classification of two-dimensional log terminal singularities (see, e.g., $\left[10\right.$, Ch. 3]). Since $b_{1}>2$, we have $\left[c_{s}-1, \ldots, c_{l}\right]=n / q$, where $1 \leq q<n, \operatorname{gcd}(n, q)=1$ and $n=2,3,4$, or 5. So, $\left[c_{s}-1, \ldots, c_{l}\right]=[n]$, $[2, \ldots, 2],[2,3]$, or $[3,2]$. 
Assume that $s=1$. Then $\left[c_{1}, \ldots, c_{l}\right]=[4]$ or $[3,3]$ (see Proposition $3.6), k=\varrho-1$, and $\left[b_{1}, \ldots, b_{k-1}\right]=n^{\prime} / q^{\prime}$, where $1 \leq q^{\prime}<n^{\prime}-$ $1, \operatorname{gcd}\left(n^{\prime}, q^{\prime}\right)=1$ and $n^{\prime}=3,4$, or 5 . Further, $\alpha_{1}^{\prime}=1 / 2$. Easy computations (see $[10,(3.1 .3)]$ ) show that $2 / b_{k}>\alpha_{k}>1 / 2$. Thus $b_{k}=3$. We get only one possibility $\left[b_{1}, \ldots, b_{k}, \ldots, b_{\varrho}\right]=[4,3,2]$. But then $\alpha_{k}=1 / 5$, a contradiction.

Assume that $s>1$. Then $c_{1}=\cdots=c_{s-1}=2$. Hence, $c_{l} \geq 3$ and $\left[c_{1}, \ldots, c_{l}\right]=[2, \ldots, 2, n+1]$, or $[2, \ldots, 2,3,3]$. Since $\left[c_{1}, \ldots, c_{l}\right]$ is a T-chain, $n \geq 4$ and $\left[c_{1}, \ldots, c_{l}\right]=[2,5]$, or $[2,2,6]$. As above we get $\alpha_{1}^{\prime} \leq 1 / 3,2 / b_{k}>\alpha_{k}>2 / 3$, and $b_{k}=2$, a contradiction.

Lemma 5.9. Notation as above. Let $D_{1}$ and $D_{2}$ be two components of $D$ such that

(i) $D_{1} \cap D_{2} \neq \varnothing$,

(ii) there are T-points $P_{i} \in D_{i}, P_{i} \notin D_{1} \cap D_{2}$.

Then $K_{S}+D_{1}+D_{2}$ is lc.

Proof. Assume the converse. By the previous lemma, $\Gamma(\varphi)$ contains a subgraph $\Gamma$ of the form

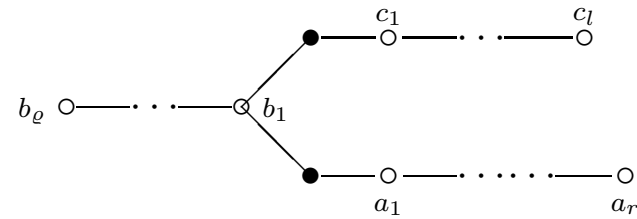

where $\varrho \geq 2$ and $\left[a_{1}, \ldots, a_{r}\right],\left[b_{1}, \ldots, b_{\varrho}\right],\left[c_{1}, \ldots, c_{l}\right]$ are T-chains.

Note that $b_{1} \geq 3$. By Corollary 3.8 and Lemma 5.3 we have $a_{1}=$ $c_{1}=2$. Take $s, m \geq 1$ so that

$$
a_{1}=\cdots=a_{s}=2, a_{s+1}>2, c_{1}=\cdots=c_{m}=2, c_{m+1}>2 .
$$

Contracting black vertices successively we get the following log terminal graph

$$
\Gamma^{\prime}=\left[b_{1}-s-m-2\left|b_{2}, \ldots, b_{\varrho}\right| a_{s+1}-1, \ldots, a_{r} \mid c_{m+1}-1, \ldots, c_{l}\right] .
$$

By Proposition 3.6 we have $\sum a_{i}=2-\beta+3 r$, where $\beta$ is the number of vertices of the corresponding chain (3.5) with $\iota=2$ ( $\beta$ coincides with $\beta(n / q)$ introduced in $\S 3$ but we do not need this fact). Since $\beta+s \leq r$,

$$
a_{s+1}+\cdots+a_{r}=2-\beta+3 r-2 s \geq 2+r+\beta \geq 5 .
$$

Similarly, $c_{m+1}+\cdots+c_{l} \geq 5$. Therefore, $\varrho=b_{\varrho}=2$ and we may assume that $\left[a_{s+1}-1, \ldots, a_{r}\right]=3 / q$. On the other hand, $a_{r} \geq 3$, so $\left[a_{s+1}-\right.$ $\left.1, \ldots, a_{r}\right]=[3]$ and $\left[a_{1}, \ldots, a_{r}\right]=[2, \ldots, 2,4]$, a contradiction.

Corollary 5.11. Let $\varphi: S \rightarrow T \ni$ o be a T-contraction. 
(i) If $S$ has exactly one non-Du Val point $P$, then all the components of $D$ pass through $P$.

(ii) If $S$ has more than one non-Du Val points, then $\left(S, D_{i}\right)$ is plt for any component $D_{i}$ of $D$ containing two non-Du Val points.

Now Theorem 1.5 is a consequence of Propositions 2.4 and 5.12 below.

Proposition 5.12. Let $\varphi: S \rightarrow T \ni o$ be a T-contraction. Then $K_{S}$ is 1-complementary.

5.13. To begin with, assume that $\varphi: S \rightarrow T \ni o$ is an arbitrary log contraction. We apply the technique developed in [16]. Take $\delta$ so that $(S, \delta D)$ is maximally $\log$ canonical.

Note that on $S$ the LMMP works with respect to any divisor $G$. Indeed, there is a boundary $F$ such that $K_{X}+F$ is klt, numerically trivial, and the components of $F$ generate $\operatorname{Pic}(S) \otimes \mathbb{Q}$. Then $G$-MMP is equivalent to $K_{S}+F+\varepsilon G^{\prime}$-MMP for $0<\varepsilon \ll 1$ and suitable $G^{\prime} \sim_{\mathbb{Q}} G$.

Lemma 5.14. Assume that $(S, \delta D)$ is plt. Then $K_{S}$ is 1complementary.

Proof. Since $(S, \delta D)$ is maximally log canonical, $\lfloor\delta D\rfloor \neq 0$. Put $C=$ $\lfloor\delta D\rfloor$ and $B=\delta D-C$. By Connectedness Lemma [17, 5.7], $C$ is an irreducible curve. By Corollary 5.6, $\operatorname{Diff}_{C}(\delta B)$ is supported in two points, say $P_{1}$ and $P_{2}$. Run $-\left(K_{S}+C\right)$-MMP over $T: \psi: S \rightarrow \bar{S}$. Since $-K_{S}$ is $\psi$-ample, $C$ is not contracted. Since $-\left(K_{S}+\delta D\right)$ is $\psi$ ample, the plt property of $(S, \delta D)$ is preserved. We get a plt model $(\bar{X}, \bar{C})$ such that $-\left(K_{\bar{S}}+\bar{C}\right)$ is nef over $T$. By the above, $\operatorname{Diff}_{\bar{C}}(\delta \bar{B})$ is supported in two points. Hence $K_{\bar{S}}+\operatorname{Diff}_{\bar{C}}$ is 1-complementary (see $[17,5.2])$. Since $-\left(K_{\bar{S}}+\bar{C}\right)$ is nef and big over $T$, this complement can be extended to $\bar{S}$ (see [15, Prop. 4.4.1]). By [15, 4.3] this gives us an 1-complement of $K_{S}+C$.

5.15. If $(S, \delta D)$ is not plt, there is an inductive blowup of $(S, \delta D)$. By definition it is a birational extraction $\sigma: S^{\prime} \rightarrow S$ with irreducible exceptional divisor $E$ satisfying the following properties:

(i) $K_{S^{\prime}}+E+\delta D^{\prime}=\sigma^{*}\left(K_{S}+\delta D\right)$ is log canonical, where $D^{\prime}$ is the proper transform of $D$,

(ii) $\left(S^{\prime}, E\right)$ is plt.

Since the minimal resolution $\mu: \tilde{S} \rightarrow S$ is a $\log$ resolution of $(S, D)$, we may assume that $\mu$ factors through $\sigma$ (see [15, Proof of 3.1.4]). Then $K_{S^{\prime}}+\alpha E=\sigma^{*} K_{S}$, where $\alpha \geq 0$ and $-\left(K_{S^{\prime}}+\alpha E\right)$ is nef over $T$. As in the proof of Lemma 5.14 we run $-\left(K_{S^{\prime}}+E\right)$-MMP over $T$. In this 
case again $E$ cannot be contracted and we get a model $(\bar{S}, \bar{E})$ such that $-\left(K_{\bar{S}}+\bar{E}\right)$ is nef over $T$ :

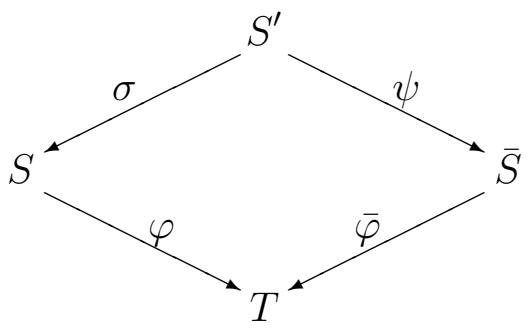

Denote by $N$ the exceptional divisor of $\psi$ and let $V:=\operatorname{Sing}\left(S^{\prime}\right) \cap E$. If $-\left(K_{S^{\prime}}+E\right)$ is nef over $T$, we put $\psi=$ id and $N=\varnothing$. Clearly all singular points $\bar{P}_{1}, \ldots, \bar{P}_{r}$ of $\bar{S}$ lying on $\bar{E}$ are contained in $\psi(V) \cup \psi(N)$. If $r \leq 2$, then $K_{\bar{E}}+\operatorname{Diff}_{\bar{E}}$ is 1-complementary (see [17]). Since $-\left(K_{\bar{S}}+\bar{E}\right)$ is nef over $T$, this complement can be extended to $\bar{S}$ (see [15, Prop. 4.4.1]). By $[15,4.3]$ this gives us an 1-complement of $K_{S}$.

Lemma 5.16. Assume that $S$ has a unique non-Du Val point and this point which is a cyclic quotient. Then $K_{S}$ is 1-complementary.

Proof. We may assume that $(S, \delta D)$ is not plt. Since $P:=\sigma(E) \in S$ is a cyclic quotient singularity, $V$ contains at most two points. Indeed, $-K_{S^{\prime}}$ is $\psi$-ample and $S^{\prime}$ has at worst $\mathrm{Du}$ Val singularities outside of $\operatorname{Sing}(\bar{S}) \cap \bar{E}$. By $[8,3.38]$ discrepancies of all divisors of $\bar{S}$ over $\psi(E)$ are strictly positive. Hence $\bar{S}$ is smooth at points of $\psi(N), \bar{P}_{1}, \ldots, \bar{P}_{r} \in$ $\psi(V)$ and $r \leq 2$. By the above $K_{S}$ is 1-complementary.

Proof of Proposition 5.12. Again $V$ contains at most two points. If $K_{S}$ is not 1-complementary, then $r \geq 3$. Take $\bar{P} \in \psi(N) \backslash \psi(V)$ and let $N_{0}=\psi^{-1}(\bar{P})$. Then the point $N_{0} \cap E \in S$ is smooth and $N_{0}$ contains at least one non-Du Val point of $S^{\prime}$. If $V$ is two points, then by Corollary 5.6 we get a subgraph (5.8), a contradiction.

Assume that $V$ is one point. Then there are two points $\bar{P}, \bar{P}_{1} \in$ $\psi(N) \backslash \psi(V)$ and similarly by Lemma 5.7 we get a subgraph (5.10), a contradiction.

Finally, assume that $S^{\prime}$ is smooth along $E$. Then $E$ is a $(-4)$-curve. Hence $\Gamma(\varphi)$ contains a fork $\left[4\left|1, b_{1}\right| 1, b_{2} \mid 1, b_{3}\right]$. Such a graph cannot be elliptic.

\section{Examples.}


Proposition 5.17. Let $\varphi: S \rightarrow T$ be a two-dimensional log conic bundle of index two. Then $\Gamma(\varphi)$ is one if the following:
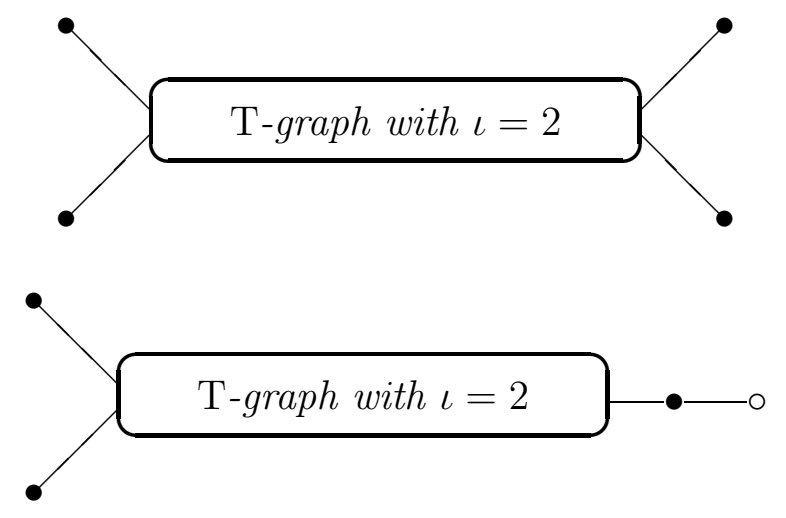

$\left(\mathrm{I}^{* * *}\right)$
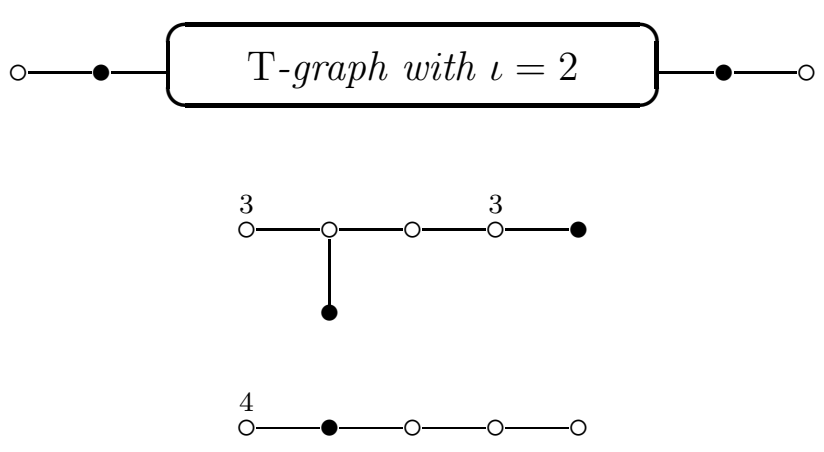

Our notation can be explained as follows. Consider a general member $B \in\left|-K_{S}\right|$ and let $S^{\prime} \rightarrow S$ be a double covering branched along $B$. Then $K_{S^{\prime}}=0, S^{\prime} \rightarrow T$ is an elliptic fibration, and $S^{\prime}$ has only Du Val singularities (cf. $[12, \S 3]$ ). If $\tilde{S}^{\prime}$ is the minimal resolution, then the central fiber of the composition map $\tilde{S}^{\prime} \rightarrow T$ is Kodaira's singular fiber.

Proof. For any index two log terminal point all log discrepancies of the minimal resolution are equal to $1 / 2$. By Lemma 5.3 there is at most one non-Du Val point on each component of $D$. By Corollary 5.6 there is exactly one non-Du Val point $P$ on $S$ and all the components of $D$ pass through $P$. Again using Lemma 5.3 we have $\sum l_{i}=4$, so the graph $\Gamma(\varphi)$ has at most four black vertices. Now the classification follows by Lemma 5.5. 
Example 5.18. The following graphs gives us examples of T-conic bundles with two and three non-Du Val points.

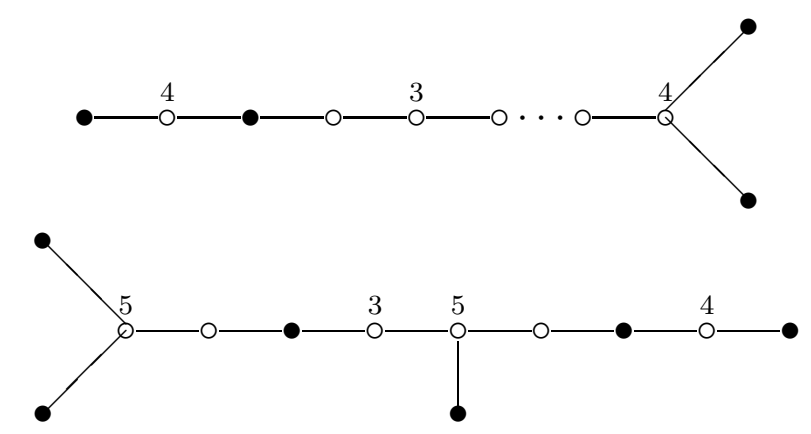

Proposition 5.19. For any T-singularity $\frac{1}{n}(1, q)$ there is a T-conic bundle having exactly one singular point which is of type $\frac{1}{n}(1, q)$.

Proof. One can start with graph $\left(\mathrm{I}^{*}\right)$ and run the construction below. By Proposition 3.6 on each step we have only singularities of type T.

Construction 5.20. Let $\varphi$ be a log conic bundle with a unique singular point that is of type $\left[b_{1}, \ldots, b_{\varrho}\right]$. Assume that in $\Gamma(\varphi)$ there is a black vertex adjacent to the end $b_{1}$, i.e., $\Gamma(\varphi)$ contains a string $\left[1, b_{1}, \ldots, b_{\varrho}\right]$, where $\left[b_{1}, \ldots, b_{\varrho}\right]$ corresponds to singular point. Blowing-up the ends 1 and $b_{\varrho}$, we get a graph $\Gamma^{\prime}$ containing a string $\left[1,2, b_{1}, \ldots, b_{\varrho}+1,2\right]$. By Remark 5.4, $\Gamma^{\prime}$ corresponds to a $\log$ conic bundle (i.e., the anticanonical divisor is ample).

Remark 5.21. (i) Construction 5.20 provides only one series of T-conic bundles with a unique singular point. For example, the following T-conic bundle cannot be obtained by this way.

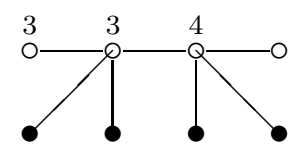

(ii) Similar to 5.20 one can obtain infinite series of T-conic bundles with two and three singular points starting with Example 5.18.

\section{The CASE OF IRREDUCiBle CENTRAL Fiber}

The following lemma shows that case of relative Picard number one is most important.

Lemma 6.1. Let $f: X \rightarrow Z \ni$ o be a Fano-Mori contraction such that the dimension of fibers is at most one. There is a Fano-Mori contraction $f^{\prime}: X^{\prime} \rightarrow Z$ with the same property and a birational map $h: X \rightarrow X^{\prime}$ over $Z$ such that $h$ is a morphism outside of $f^{-1}(o), X^{\prime}$ 
is $\mathbb{Q}$-factorial and $\rho(X / Z)=1$. In particular, $f^{\prime-1}(L)$ is irreducible for any irreducible divisor $L \subset Z$. If furthermore $\left(X, f^{*} T\right)$ is dlt for some effective Cartier divisor $T$, then we can take $X^{\prime}$ so that $\left(X^{\prime}, f^{\prime *}(T)\right)$ is $d l t$.

Proof. Let $q: X^{\mathrm{q}} \rightarrow X$ be a $\mathbb{Q}$-factorial modification. Thus $X^{\mathrm{q}}$ has only terminal $\mathbb{Q}$-factorial singularities, $K_{X^{q}}=q^{*} K_{X}$, and $q$ is a small birational contraction. Run MMP over $Z$. We get $X^{\prime}$ as above.

To show the second statement we construct $\mathbb{Q}$-factorialization $q: X^{\mathrm{q}} \rightarrow X$ of $(X, S)$. Then $\left(X^{\mathrm{q}}, S^{\mathrm{q}}\right)$ is dlt, where $S^{\mathrm{q}}=q^{*} S$. Then we just note that $K_{X^{q}}-\mathrm{MMP}$ is the same as $K_{X^{\mathrm{q}}}+S^{\mathrm{q}}-\mathrm{MMP}$.

In analytic situation $\rho(X / Z)=1$ implies that the fiber $f^{-1}(o)$ is irreducible. Now we classify semistable Mori conic bundles with irreducible central fiber.

Proposition 6.2 (cf. [14, Th. 2.5]). Let $\varphi: S \rightarrow T \ni$ o be a T-conic bundle having at least one non-Du Val point. Assume that the fiber $D$ is irreducible. Then $\varphi$ is of type (III*) of 5.17 .

Corollary 6.3. Let $f: X \rightarrow Z \ni$ o be a semistable Mori conic bundle such that $f^{-1}(o)$ is irreducible. Then $X$ has exactly one non-Gorenstein point which is of index 2 , see [12, §3].

Proof. If $K_{S}+C$ is plt, then $S$ has two singularities of types $\frac{1}{n}(1, q)$ and $\frac{1}{n}(1, n-q)$ (see [14, Th. 2.5]). If they are of type T, then

$$
(q+1)^{2} \equiv 0 \quad \bmod n, \quad(n-q+1)^{2} \equiv 0 \quad \bmod n .
$$

This gives us $4 \equiv 0 \bmod n$. Since the singularities of $S$ are worse than Du Val, $n=4$. We get case (III*).

Now we consider the case when $K_{S}+C$ is not plt. By Lemma 5.7 and Corollary 5.6 the surface $S$ has exactly one non-Du Val point and at most one Du Val point $Q$. Moreover, $S \ni Q$ is of type $A_{n}$ and $K_{S}+C$ is plt near $Q$. Thus $\Gamma(\varphi)$ has the following form

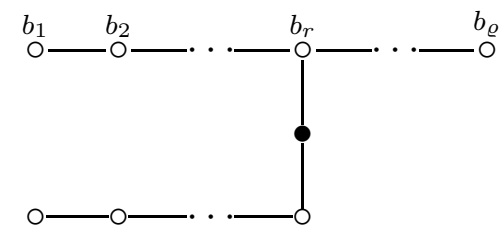

where $r \neq 1, r \neq \varrho$. Contracting black vertices successively, on some step we get a subgraph

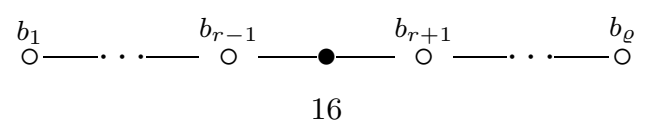


Lemma 6.6. If the graph (6.5) is parabolic, then

$$
\sum_{i=1}^{r-1}\left(b_{i}-1\right)=\sum_{j=r+1}^{\varrho}\left(b_{j}-1\right)=\varrho-2 .
$$

In particular, $r \neq 1, \varrho$.

Apply the procedure described in Proposition 3.6 to $\left[b_{1}, \ldots, b_{r}, \ldots, b_{\varrho}\right]$. Each step preserves relation (6.7). At the end we get a chain $\left[b_{1}^{\prime}, \ldots, b_{r^{\prime}}^{\prime}, \ldots, b_{\varrho^{\prime}}^{\prime}\right]$ as in (3.5). Relation (6.7) give us $r^{\prime}=\varrho^{\prime}-r^{\prime}+1=\varrho^{\prime}-2$, i.e., $r^{\prime}=3$ and $\varrho^{\prime}=5$. Hence, in (6.5) we have $b_{r-1}=b_{r+2}=2$. This contradicts Lemma 5.5.

\section{The EXISTEnCE OF SEMISTABLE 3-FOLD FLIPS}

Theorem 7.1. Let $f: X \rightarrow Z$ be a semistable three-dimensional flipping contraction. Assume that $f$ is extremal (i.e., $X$ is $\mathbb{Q}$-factorial and $\rho(X / Z)=1)$. Then the flip of $f$ exists.

Sketch of the proof $($ see $[4,8.5,8.7])$. The existence of the flip is equivalent to the finite generation of the $\mathcal{O}_{Z}$-algebra $\mathcal{R}_{Z}\left(K_{Z}\right):=$ $\oplus_{m \geq 0} \mathcal{O}_{Z}\left(m K_{Z}\right)$, see [4, Lemma 3.1]. By Theorem 1.5 there is $L=$ $2 F \in\left|-2 K_{X}\right|$ such that $K_{X}+f^{*} T+\frac{1}{2} L$ is lc. Since $f$ is an extremal contraction and $K_{X}+f^{*} T+\frac{1}{2} L$ is numerically trivial, one can see that $K_{Z}+T+\frac{1}{2} L_{Z}$ is also lc, where $L_{Z}:=f_{*} L \in\left|-2 K_{Z}\right|$. Therefore, the same holds for a general member $L_{Z} \in\left|-2 K_{Z}\right|$ which is reduced and irreducible. As in $[4, \S 8]$, consider a double covering $\pi: Z^{\prime} \rightarrow Z$ ramified along $L_{Z}$. Then $K_{Z^{\prime}}+\pi^{*} T=\pi^{*}\left(K_{Z}+T+\frac{1}{2} L_{Z}\right)$ is lc and Cartier. Since $\pi^{*} T$ also is a Cartier divisor, $Z^{\prime}$ has at worst a canonical Gorenstein singularity at $o^{\prime}:=\pi^{-1}(o)$. Put $L_{Z^{\prime}}:=\pi^{*}\left(L_{Z}\right)_{\text {red. }}$. By [4, 3.2] the finite generation of algebras $\mathcal{R}_{Z}\left(K_{Z}\right)$ and $\mathcal{R}_{Z^{\prime}}\left(K_{Z}^{\prime}-L_{Z}^{\prime}\right)$ is equivalent. Finally, the last algebra is finitely generated by $[4,6.1]$ (see also $[6],[10, \S 6],[8, \S 6])$.

Note that in our case the finite generation of $\mathcal{R}_{Z^{\prime}}\left(K_{Z}^{\prime}-L_{Z}^{\prime}\right)$ is much easier because the presence of a Cartier divisor $\pi^{*} T$ such that $K_{Z}^{\prime}+\pi^{*} T$ is lc.

Corollary 7.2 ([7]). Let $f: X \rightarrow Z \ni$ o be a semistable birational contraction with fibers of dimension at most one (either flipping or divisorial of type 2-1) and let $T$ be a general hyperplane section through o. Then $T \ni o$ is a cyclic quotient singularity. If furthermore $f$ is divisorial, then $T \ni o$ is of type $\mathrm{T}$. 
Proof. By Theorem 1.5 the pair $(Z, T+f(F))$ is log canonical. By Adjunction so is $\left(T, \operatorname{Diff}_{T}(f(F))\right)$. Moreover, $K_{T}+\operatorname{Diff}_{T}(f(F)) \sim 0$. By the classification of log terminal singularities with a reduced boundary $T \ni o$ is a cyclic quotient singularity.

\section{REFERENCES}

[1] Corti A. Semistable 3-fold flips, e-print alg-geom/9505035

[2] Looijenga E. \& Wahl J. Quadratic functions and smoothing surface singularities, Topology 25 (1986) 261-291

[3] Mori S. Flip theorem and the existence of minimal models for 3-folds, J. Amer. Math. Soc. 1 (1988) 117-253

[4] Kawamata Y. Crepant blowing-up of 3-dimensional canonical singularities and its application to degenerations of surfaces, Ann. Math. (2) 127 (1988) 93-163

[5] Kawamata Y. Semistable minimal models of threefolds in positive or mixed characteristic, J. Algebraic Geom. 3 (1994) 463-491

[6] Kollár J. Flops, Nagoya Math. J. 113 (1989) 15-36

[7] Kollár J. \& Mori S. Classification of three-dimensional flips, J. Amer. Math. Soc. 5 (1992) 533-703

[8] Kollár J. \& Mori S. Birational geometry of algebraic varieties. Cambridge Tracts in Mathematics, 134. Cambridge University Press, Cambridge, 1998

[9] Kollár J. \& Shepherd-Barron N. I. Threefolds and deformations of surface singularities, Invent. Math. 91 (1988) 299-338

[10] Kollár J. et al. Flips and abundance for algebraic threefolds, in "A summer seminar at the Univ. of Utah, Salt Lake City, 1991". Astérisque. 211 (1992)

[11] Kulikov Vik. S. Degenerations of K3 surfaces and Enriques surfaces, Izv. Akad. Nauk SSSR Ser. Mat. 41 (1977) 1008-1042

[12] Prokhorov Yu. G. On the existence of complements of the canonical divisor for Mori conic bundles, Russian Acad. Sci. Sb. Math. 188 (1997) 1665-1685

[13] Prokhorov Yu. G. On extremal contractions from threefolds to surfaces: The case of one nonsingular point, Contemporary Math. AMS 207 (1997) 119-141

[14] Prokhorov Yu. G. Mori conic bundles with a reduced log terminal boundary, J. Math. Sci. (New York) 94 (1999) 1051-1059

[15] Prokhorov Yu. G. Lectures on complements on log surfaces. MSJ Memoirs. 10. Tokyo: Mathematical Society of Japan. (2001)

[16] Prokhorov Yu. G. \& Shokurov V. V. The first main theorem on complements: from global to local, Russian Acad. Sci. Izv. Math. 65 (2001) 1169-1196

[17] Shokurov V. V. 3-fold log flips, Russ. Acad. Sci. Izv. Math. 40 (1993) 93-202

[18] Shokurov V. V. Semistable 3-fold flips, Russ. Acad. Sci. Izv., Math. 42 (1994) $371-425$

[19] Tsunoda Sh. Degenerations of surfaces, Algebraic geometry, Sendai, 1985, 755-764, Adv. Stud. Pure Math., 10, North-Holland, Amsterdam, 1987

Department of Algebra, Faculty of Mathematics, Moscow State University, Moscow 117234, Russia

E-mail address: prokhoro@mech.math.msu.su 